\title{
Syringomyelia in the Thoracolumbar Spinal Cord of an African Wild Dog (Lycaon Pictus)
}

\author{
*Jessica M Weeks BS ${ }^{1}$, 2,6Jennifer N Langan, DVM Dipl ACZM, ${ }^{3}$ Hylton Gelb DVM Dipl ACVR, ${ }^{4}$ Benjamin N \\ Nevitt DVM, ${ }^{5}$ Sarah M Corner DVM Dipl ACVP and ${ }^{6}$ Michael J Adkesson DVM Dipl ACZM \\ ${ }^{1}$ University of Florida College of Veterinary Medicine, USA
}

${ }^{2}$ Department of Veterinary Clinical Medicine, College of Veterinary Medicine, University of Illinois, USA

${ }^{3}$ Veterinary Specialty Center, USA

${ }^{4}$ Illinois Zoological and Aquatic Animal Residency Program, University of Illinois, College of Veterinary Medicine, USA

${ }^{5}$ Zoological Pathology Program, University of Illinois, USA

${ }^{6}$ Chicago Zoological Society, USA

Received: September 22, 2017; Published: October 10, 2017

*Corresponding author: : Jennifer Langan, Department of Veterinary Clinical Medicine, College of Veterinary Medicine, University of Illinois, 2001 South Lincoln Avenue, Urbana, Illinois, 61802, USA

\begin{abstract}
A captive born neonatal African wild dog (Lycaon pictus) exhibited hind limb weakness and ataxia since the onset of ambulation. Initial examination at one month of age revealed postural reaction deficits in both hind legs and moderate muscle atrophy. After sustaining a bite wound to the head, the animal was reexamined at 87 days of age. A cerebrospinal fluid analysis showed a mild mononuclear pleocytosis. Magnetic resonance imaging indicated a large syrinx in the thoracolumbar spinal cord. The animal was subsequently euthanized and necropsy confirmed the presence of a $1.5 \mathrm{~cm}$ x $3 \mathrm{~mm}$ syrinx at the T13-L1 level of the spinal cord. This is the first report of syringomyelia in any nondomestic canid. Limited genetic diversity in the captive African wild dog population may contribute to increasing prevalence of rare congenital and inherited diseases, which could support strict breeding management in this species.
\end{abstract}

Keywords: African Wild Dog; Canine; Congenital Malformation; Lycaon pictus; MRI; Syringomyelia

\section{Brief Communication}

A 31-day-old, captive born, male, African wild dog (Lycaon pictus, AWD) displayed hind limb ataxia and weakness since the onset of ambulation. Separation of the animal carried a high risk of aggression to the litter from the pack, leading to a conservative medical approach throughout the case. At 36 days of age, the clinical signs became more notable and the animal was visually smaller and thinner than its littermates. Although able to stand, it displayed an unsteady gait, taking a few steps at a time before the hind limbs collapsed. On physical examination, the animal was bright, alert and responsive, had a body condition score of $2 / 5$, and displayed postural reaction (PR) deficits in both hind legs, more pronounced on the left. The remainder of the neurological examination, including the thoracic limb reflexes and cranial nerve evaluation, was normal. Ulcerated skin calluses of $1 \mathrm{~cm}$ diameter were present bilaterally on the elbows as a result of the hind limb weakness and increased time spent in recumbency. Mild laxity in the coxofemoral joints was noted on palpation. Radiographs showed left coxofemoral joint subluxation and mild muscle atrophy of the left hind limb. There was also asymmetry of the left capital femoral physis. A computed tomography (CT) scan was performed (HiSpeed CT/I, GE Medical Systems, Waukesha, Wisconsin 53188, USA; 3mm slice thickness, $1.25 \mathrm{~mm}$ reconstruction interval, soft tissue reconstruction algorithm) that supported the findings of the left hind limb muscle atrophy, but could not confirm the coxofemoral joint changes seen on radiographs. No abnormalities of the vertebral column or spinal cord were detected. The gait abnormalities were thought to be due to laxity in the tendons and ligaments of the coxofemoral joints.

As the animal grew, its ability to ambulate improved, but the gait remained abnormal with the animal intermittently falling to the side with both hind limbs. At 45 days of age it was able to keep up with the pack and by 52 days the elbow lesions were resolving. At 66 days of age, the animal was reevaluated. Body condition score had declined to $1.5 / 5$ and new superficial abrasions were present on the dorsal lateral aspect of both hind feet, due to its abnormal 
ambulation. Significant muscle atrophy and marked ataxia in the hind end were noted. The animal was able to rise to a standing position and ambulate, but would drag its hind legs more often than previously observed, especially at a run. PR was now completely absent in both pelvic limbs. No pain was elicited on spinal or neck palpation, and deep pain was present bilaterally in the hind feet. Mentation was still normal, thoracic reflexes were normal, and anal tone was present. The animal was suspected to have a spinal lesion caudal to third thoracic vertebrae (T3), given a guarded prognosis, and returned to the litter with close monitoring of quality of life.

The gait abnormalities progressively worsened as the littermates were maturing and integrating into the pack. On day 87 the animal sustained a bite wound to the cranium, prompting intervention for treatment and further assessment. Cranial nerve examination and thoracic limb reflexes were normal, and mentation remained appropriate, but the animal was now paraparetic. Multiple superficial abrasions were noted on the elbows, hocks, and feet. Cerebrospinal fluid (CSF) was collected from the L5-6 intervertebral space. Culture revealed no growth of aerobic or anaerobic organisms. Fluid analysis showed a mild mononuclear pleocytosis (total nucleated cell count $(\mathrm{TNCC})=5$ cells/uL, 58\% large mildly vacuolated mononuclear cells, 42\% small lymphocytes), a mild increase in protein $(61.7 \mathrm{mg} / \mathrm{dL})$, and peripheral blood contamination (586 red blood cells (RBC)/uL). Normal canine reference intervals for this laboratory include: TNCC $<5$ cells/uL, protein concentration $<45 \mathrm{mg} / \mathrm{dL}$ for lumbar samples, and $>500$ RBCs/uL was considered peripheral blood contamination. Findings were considered non-specific and typical of inflammation, infection, or trauma to the central nervous system.

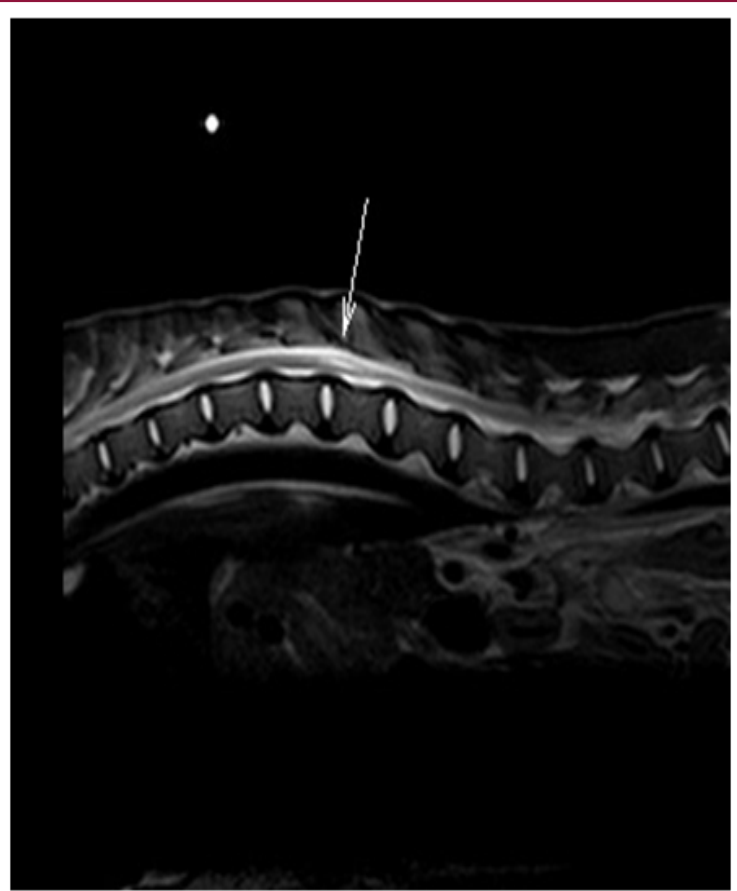

Figure 1: Sagittal T2-weighted magnetic resonance image showing hyperintensity within the spinal cord representing syrinx formation at the level of T13-L1 (arrow).
A magnetic resonance imaging (MRI) scan (Philips Gyroscan NT, Imaging Center for Animals, Buffalo Grove, IL 60089, USA) showed a non-contrast enhancing, T2-weighted hyperintensity within the central and dorsal aspect of the right spinal cord extending from the level of the caudal aspect of T13 caudally to the level of L1-L2 (Figure 1). There was also mild bulging of the L7-S1 intervertebral disc without significant compression. The lesion visualized via MRI and the progressive worsening of clinical signs support the diagnosis of syringomyelia of the thoracolumbar spinal cord. Surgical intervention was not pursued due to the extensive postoperative care and aggressive social behavior of AWD. Mounting concern for the pack injuring or killing this debilitated member prompted euthanasia.

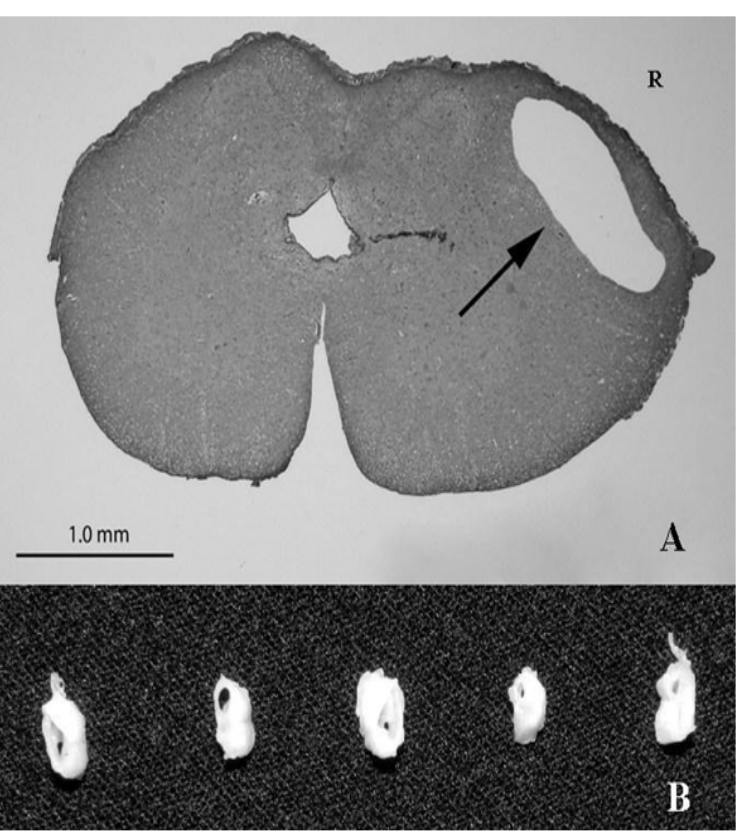

Figure 2: (A) Histological section, spinal cord, T13. The image is not the most affected portion of the spinal cord, but demonstrates the syrinx clearly. The right dorsal and lateral funiculi contain a well-demarcated cavitation, or syrinx, delineated by mildly compressed neuropil (arrow). The syrinx does not communicate with the central canal. The central canal is mildly ectatic. " $R$ " indicates right side. Hematoxylin and Eosin.

(B) Gross spinal cord, T13-L1. Multiple cross sections of T13 to L1 spinal cord have a variable sized cavitation within the gray and white matter. This cavitation is 1.5 $\mathrm{cm}$ long and up to $3 \mathrm{~mm}$ diameter. Fixed sections $(10 \%$ buffered formalin).

On necropsy, the animal had adequate subcutaneous and visceral adipose tissue stores. Epaxial, gluteal and hind limb skeletal muscles were markedly atrophied. Bilaterally, skin overlying the olecranon processes of the elbows was ulcerated and overlain by a serocellular crust, interpreted as pressure sores. A $1.5 \mathrm{~cm}$ long, up to $3 \mathrm{~mm}$ diameter round to irregular cavitation, or syrinx, in the T13-L1 spinal cord was identified that markedly compressed and distorted the adjacent neuropil. On histopathology, the T13-L1 spinal cord had a well-demarcated cavitation, or syrinx, involving the dorsal, lateral and ventral funiculi white matter as well as 
adjacent grey matter (Figure 2). The syrinx was circumferentially demarcated by mildly compressed neuropil. Adjacent axon sheaths were dilated and empty, or contained swollen spheroids, which indicate axonal loss and degeneration, respectively. A few empty axon sheaths contained a single gitter cell. The bulging disc noted via MRI was not appreciated grossly. The necropsy findings confirmed the diagnosis of syringomyelia.

Syringomyelia is a general term that characterizes a spinal cord cavitation containing fluid [1]. Syringomyelia is a secondary finding due to a primary cause. A cavitation in the spinal cord, or a syrinx, has been associated with primary disorders including spinal cord tumors, myelitis, meningitis, congenital vascular malformations, chronic arachnoiditis, and spinal cord trauma [1]. The cause of syringomyelia is unknown, but may result from venous obstruction, mechanical disruption, or shearing of spinal cord tissue planes.

Clinical signs associated with syringomyelia depend on lesion location within the spinal cord [2]. In this case, the hind limb paresis, weakness, and lack of normal postural reactions of the pelvic limbs, yet normal forelimb reflexes were consistent with deficits in the sensory and/or motor components of the central nervous system, and localized the lesion to the T3-L3 spinal cord segment [3]. While the diagnosis of the large syringomyelia at the T13-L1 spinal cord was deemed the cause of the gait abnormalities, the size of the syrinx determined by MRI does not always correlate with severity of clinical signs [4]. Cavitation lesions may be progressive and expand along the gray matter of the dorsal horns, which are planes of structural weakness, resulting in subsequent necrosis and edema of the spinal cord parenchyma around the cavitation and progression of clinical signs [2]. In this case, clinical signs progressed as hind limb PR was delayed at one month of age and absent by three months of age.

Syringomyelia has been documented in humans [4], domestic dogs [2,5,6], domestic cats [7], cattle [8], horses [9], an African lion [10], and a dromedary camel [11]. To the authors' knowledge, this is the first report of syringomyelia in any non-domestic canid. Previously thought to be a rare condition, diagnosis of syringomyelia in domestic companion animals is now more common, especially with the increased availability of MRI. This disorder is well described in certain dog breeds, especially the Cavalier King Charles spaniel [1]. In dogs, Chiari-like malformations are the most commonly described cause of syringomyelia, which typically occurs at the craniocervical junction. Syringomyelia caudal to the cervical spinal cord has been associated with spinal dysraphism [5], vertebral malformations, intervertebral disc disease and arachnoid cysts [6].

Diagnosis of syringomyelia is commonly made via breed disposition, clinical signs, MRI findings, and CSF analysis. When syringomyelia is detected via MRI, the primary cause should be sought out both cranial and caudal to the lesion [12]. MRI in this case showed a mild bulging of the L7-S1 intervertebral disc, caudal to the spinal lesion. The intramedulary pulse pressure theory suggests syringomyelia can develop a distance from the obstruction of CSF flow in any part of the spinal cord [1], and a bulging disc could have narrowed the diameter of the vertebral canal enough to alter the CSF fluid dynamics. The MRI did not show significant compression of the spinal cord from the bulging L7-S1 intervertebral disc, making it a likely incidental finding and not the cause of the syrinx formation.

While MRI is the best diagnostic tool, collection of CSF can indicate the degree of the spinal cord involvement. In a study of dogs diagnosed with Chiari-like malformations, CSF of dogs with syringomyelia had higher total nucleated cell count, higher protein concentration, and an increased neutrophil percentage. There was also a strong positive correlation between total nucleated cell count and the size of the syrinx [13]. Disruption of the blood-spinal cord barrier resulting in increased permeability has been suggested as the most likely mechanism of increased CSF protein concentration with syringomyelia. In this case, there was a mild increase in total nucleated cells and protein concentration.

Medical management of syringomyelia is directed towards analgesia, corticosteroids, and drugs that decrease CSF production. Surgical intervention is indicated when neurologic deficits are present, with the intent of restoring CSF dynamics by addressing the primary cause [6]. Direct draining of the syrinx with stents or shunts does not carry a good long term outcome in people due to obstructions [1]. Surgery was indicated for this AWD based on the severity of the clinical signs, but the extensive post-operative care would have prevented re-introduction to the pack and was not deemed in the animal's best welfare.

Syringomyelia associated with Chiari-like malformations in Cavalier King Charles spaniels is a complex trait that has a moderately high heritability [14]. Although the syrinx that occurred in this AWD was not due to a Chiari-like malformation, it is possible that the primary malformation is heritable. Decreasing genetic diversity is expected to increase the frequency of congenital and heritable diseases. The AWD population is endangered and has experienced a significant loss of genetic diversity in the managed population, which is contributing to development of a genetic bottleneck for this species [15]. At present, there is insufficient evidence from this single case to suggest syringomyelia is a heritable condition in this species, but this should be closely monitored in populations under professional care. This report highlights the importance of species specific breeding management, and the need for careful planning to maximize the genetic diversity and minimize potential of genetically heritable diseases.

\section{Acknowledgment}

The authors would like to thank the veterinary technicians and animal care staff from Chicago Zoological Society's Brookfield Zoo, as well as Drs. Belinda Comito, Devon Hague and Paul West for their assistance with this case.

\section{References}

1. Rusbridge C, D Greitz, BJ Iskander (2006) Syringomyelia: current concepts in pathogenesis, diagnosis and treatment. J Vet Intern Med 20(3): 469-479.

2. LeCouteur RA, JL Grandy (2005) Diseases of the spinal cord. In: Ettinger 
SJ, EC Feldman (Eds.), Textbook of Veterinary Internal Medicine $\left(6^{\text {th }}\right.$ edn). pp. 842-887.

3. Schatzberg SJ (2010) Neurologic examination and neuroanatomic diagnosis. In: Ettinger SJ, EC Feldman (Eds.), Textbook of Veterinary Internal Medicine, $\left(7^{\text {th }}\right.$ edn $)$. Elsevier Saunders, St Louis, USA, pp. 14011412 .

4. Magge SN, MD Smyth, LS Governale, L Goumnerova, J Madsen, et al. (2011) Idiopathic syrinx in the pediatric population: a combined center experience. J Neuros-Pediatr 7: 30-36.

5. Furneaux RW, CE Doige, MM Kaye (1973) Syringomyelia and spina bifida occulta in a Samoyed dog. Can Vet J 14: 317-321.

6. Oxley W, J Pink (2012) Amelioration of caudal thoracic syringohydromyelia following surgical management of an adjacent arachnoid cyst. J Small Anim Pract 53(1): 67-72.

7. Kitagawa M, M Okada, T Sato, K Kanayama, T Sakai (2007) A feline case of isolated fourth ventricle with syringomyelia suspected to be related with feline infectious peritonitis. J Vet Med Sci 69(7): 759-762.

8. Ohfugi S (1999) Spinal dysraphism in a newborn Holstein-Friesian calf. Vet Pathol 36(6): 607-609.
9. Hamir AN (1995) Syringomyelia in a stallion. Vet Rec 137(12): 293-294.

10. McCain S, M Souza, E Ramsay, J Schumacher, S Hecht, et al. (2008) Diagnosis and surgical treatment of a Chiari I-like malformation in an African lion (Panthera leo). J Zoo Wildl Med 39(3): 421-427.

11. Sakamoto K, M Kiupel, N Frank, PA (2004) Vertebral malformation, syringomyelia, and ventricular septal defect in a dromedary camel (Camelius dromedarius). J Vet Diagn Invest 16(4): 337-340.

12. Kitagawa M, H Ueno, SWatanabe, O Igarashi, Y Uzuka, et al. (2008) Clinical improvement in two dogs with hydrocephalus and syringohydromyelia after ventriculoperitoneal shunting. Aust Vet J 86(1-2): 36-42.

13. Whittaker DE, K English, IM McGonnell, HA Volk (2011) Evaluation of cerebrospinal fluid in Cavalier King Charles Spaniel dogs diagnosed with Chiari-like malformation with or without concurrent syringomyelia. J Vet Diagn Invest 23(2): 302-307.

14. Lewis T, C Rusbridge, P Knowler, S Blott, JA Woolliams (2010) Heritability of syringomyelia in Cavalier King Charles spaniels. Vet J 183(3): 345-347.

15. Marsden CD, H Verberkmoes, R Thomas, RK Wayne, BK Mable (2013) Pedigrees, MHC and microsatellites: an integrated approach for genetic management of captive African wild dogs (Lycaon pictus). Conserv Genet 14(1): 171-183.

BIOMEDICAL
RESERCHES $\quad \begin{aligned} & \text { Assets of Publishing with us } \\ & \text { - Global archiving of articles }\end{aligned}$

